\title{
Pathological Complete Response of Clinical T4b Ascending Colon Cancer after Preoperative Chemotherapy Using Pembrolizumab
}

\author{
Terufumi Yoshida $^{a}$ Hisanori Miki ${ }^{a}$ Hironaga Satake ${ }^{b}$

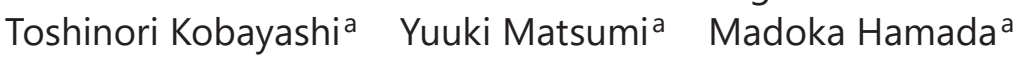 \\ Shogen Boku ${ }^{b}$ Nobuhiro Shibata ${ }^{b}$ Mitsuaki Ishida ${ }^{c}$ Mitsugu Sekimoto ${ }^{a}$ \\ ${ }^{\text {aD }}$ epartment of Surgery, Kansai Medical University Hospital, Hirakata, Japan; ${ }^{\text {bC Cancer }}$ \\ Treatment Center, Kansai Medical University Hospital, Hirakata, Japan; 'Department of \\ Pathology and Diagnostics, Kansai Medical University Hospital, Hirakata, Japan
}

Keywords

Pembrolizumab - Preoperative chemotherapy · Colon cancer - Microsatellite instability-high . $\mathrm{dMMR} \cdot$ Complete response

\begin{abstract}
Preoperative chemotherapy is efficacious in several cancers. However, it is not an established treatment for locally advanced colon cancer, particularly cases with microsatellite instabilityhigh (MSI-H)/deficient mismatch repair. Herein, we report a case of pathological complete response of MSI-H clinical T4b ascending colon cancer to preoperative treatment with pembrolizumab. A 78-year-old man was diagnosed with ascending colon cancer that invaded into the iliacus muscle and enlarged regional lymph nodes. The tumor was classified as T4bN1bM0 stage IIIC according to the 8th Union for International Cancer Control guidelines, with MSI-H status. Based on our initial diagnosis, this tumor could not be resected completely. Thus, the patient underwent preoperative therapy with CAPOX (capecitabine and oxaliplatin combination) plus bevacizumab. After 4 cycles of preoperative CAPOX/bevacizumab, we observed tumor reduction corresponding to a partial response based on the Response Evaluation Criteria in Solid Tumors criteria. Nevertheless, tumor invasion of the iliacus muscle persisted. Since oxaliplatin-induced peripheral sensory neuropathy was observed, we discontinued treatment with oxaliplatin and changed the regimen to pembrolizumab in anticipation of the therapeutic effect of this immune checkpoint inhibitor against MSI-H tumors. After 2 cycles of therapy with pembrolizumab (200 mg/body on day 1 every 3 weeks), there was drastic
\end{abstract}


tumor regression. In addition, computed tomography indicated that all lymph node metastases had disappeared. Therefore, the patient underwent laparoscopic right hemicolectomy with D3 lymph node dissection. Analysis of the resected specimen showed pathological complete response.

\section{Introduction}

Locally advanced colon cancer (LACC) is defined as a primary colon cancer with direct invasion of adjacent structures or extensive regional lymph node involvement. Extensive en bloc resection is required in LACC to achieve negative microscopic margins. Despite this aggressive approach, the rate of R0 resections remains unsatisfactory at 40-90\% [1], while the 5-year survival ranges from $28 \%$ to $73 \%$. Furthermore, the rates of postoperative morbidity and mortality are high due to requirement for multivisceral resection $[1,2]$. The usefulness of preoperative chemotherapy has not been assessed in LACC [3]. In this case report, we show that preoperative chemotherapy with pembrolizumab and radical surgery is an extremely effective regimen in LACC with microsatellite instability-high (MSI-H), resulting in an impressive pathological complete response (pCR).

\section{Case Report}

A 78-year-old man presented with a positive fecal occult blood test. Laboratory findings revealed slight anemia and a normal range of tumor markers (hemoglobin: $10.0 \mathrm{~g} / \mathrm{dL}$, carcinoembryonic antigen [CEA]: $1.0 \mathrm{ng} / \mathrm{mL}$, and carbohydrate antigen 19-9: $19.7 \mathrm{U} / \mathrm{mL}$ ). Colonoscopy revealed a circumferential type 3 tumor at the ascending colon (Fig. 1). Biopsy revealed a moderately differentiated adenocarcinoma with wild-type $R A S, B R A F^{V 600 \mathrm{E}}$ mutation, and MSI-H. Enhanced CT detected a tumor (tumor size: $43 \times 45 \mathrm{~mm}$ ) at the ascending colon which appeared to infiltrate the iliacus muscle; this was accompanied by swelling of regional lymph nodes (Fig. 1). There was no evidence of distant metastasis. Based on these findings, we reached a clinical diagnosis of ascending colon cancer T4bN1bM0 stage IIIC according to the guidelines established by the 8th Union for International Cancer Control. Considering the possible extensive invasion of structures surrounding the tumor, we concluded that resection of the tumor (even extensive) would be unsuccessful. Therefore, we recommended preoperative chemotherapy followed by radical surgery for this patient.

After 4 cycles of treatment with CAPOX plus bevacizumab (capecitabine: 1,000 mg/m $\mathrm{m}^{2}$ twice daily on days 1-14; oxaliplatin: $130 \mathrm{mg} / \mathrm{m}^{2}$ on day 1 ; and bevacizumab: $7.5 \mathrm{mg} / \mathrm{kg}$ on day 1, every 3 weeks), the tumor showed a partial response to treatment based on the Response Evaluation Criteria in Solid Tumors, version 1.1 (i.e., a decrease of $\geq 30 \%$ in tumor size) (Table 1). However, the level of CEA was elevated to $10.1 \mathrm{ng} / \mathrm{mL}$. According to the Common Terminology Criteria for Adverse Events, we observed grade 2 peripheral sensory neuropathy caused by oxaliplatin; hence, we determined that it would be difficult to continue therapy with oxaliplatin. Six weeks after the last cycle of treatment with CAPOX plus bevacizumab, the regimen was changed to pembrolizumab (dose: 200 mg every 3 weeks) in anticipation of the therapeutic effect of this immune checkpoint inhibitor against tumors with MSI-H status. After 2 cycles of therapy with pembrolizumab, the concentration of CEA was reduced to $3.1 \mathrm{ng} / \mathrm{mL}$. CT performed 1 week after the last cycle with pembrolizumab revealed

\section{Karger'}



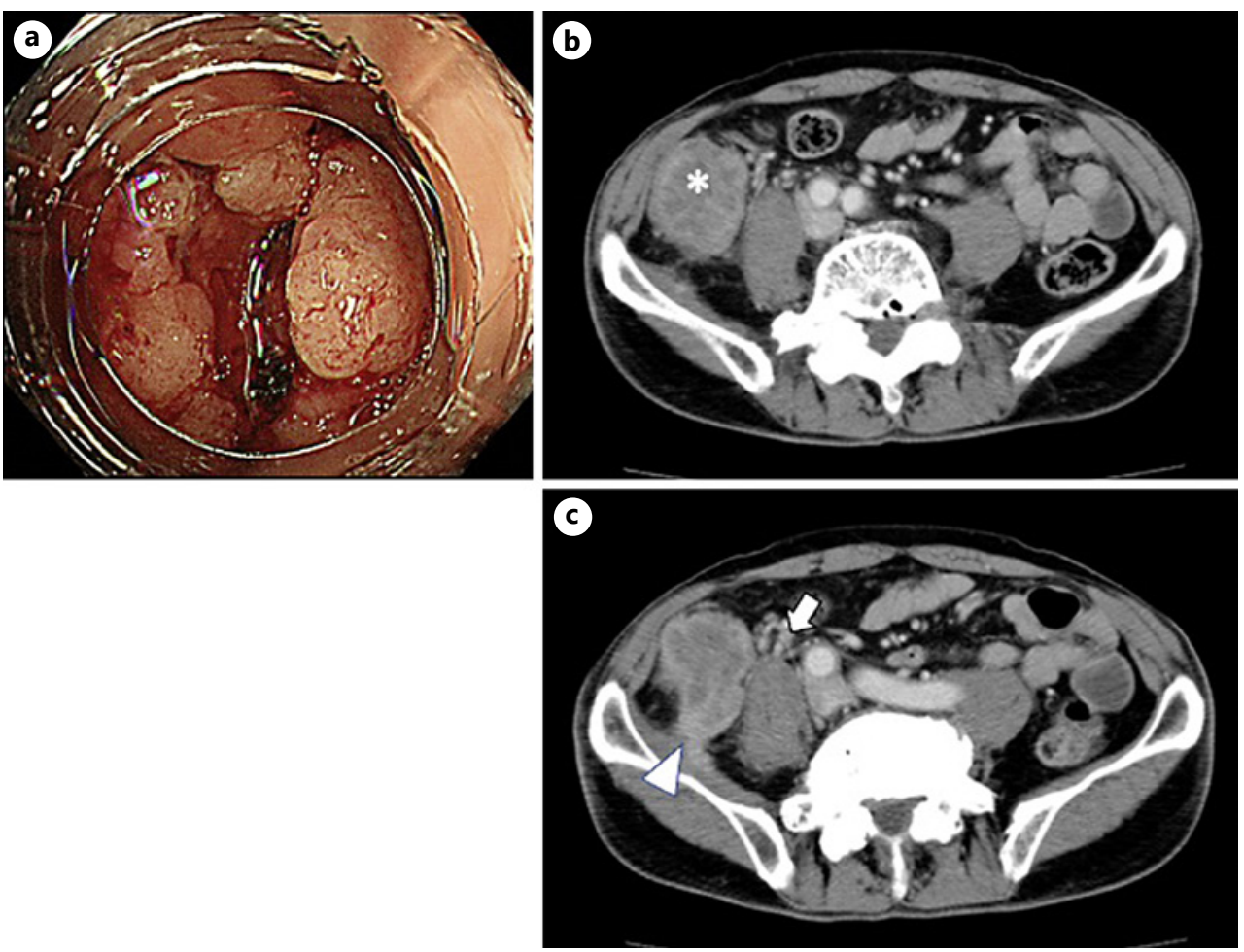

Fig. 1. Colonoscopy (a). A circumferential type 3 tumor was identified at the ascending colon. CT: a tumor (tumor size: $43 \times 45 \mathrm{~mm}$ ) was identified at the ascending colon (b). Regional lymph nodes were swollen (arrow), and the tumor infiltrated the iliacus muscle (arrowhead) (c). CT, computed tomography.

remarkable tumor regression and absence of swollen lymph nodes (Table 1). Moreover, there was no fluorodeoxyglucose uptake on positron emission tomography-CT (Fig. 2). Nevertheless, residual tumor remained, and invasion of the iliacus muscle persisted, though the invasion area was narrow (Table 1). Based on these findings, we reached a clinical diagnosis of ycT4bN0M0 stage IIC and deemed the tumor resectable. We performed radical surgery consisting of laparoscopic right hemicolectomy with D3 lymph node dissection and shaving of the iliacus muscle. Pathological analysis revealed absence of residual cancer cells and mucus accumulation at the submucosal to subserosa layer in the resected specimen (Fig. 3). Pretreatment biopsies were negative for programmed cell death 1 (PD-1) and programmed cell death-ligand 1 (PD-L1) (Fig. 4). CD8 ${ }^{+}$cells were present in the tumor tissue collected prior to treatment. However, there was no significant difference in the number of $\mathrm{CD}^{+}$cells between posttreatment surgical specimens and pretreatment biopsies (Fig. 4). The final diagnosis was pCR, ypT0, ypN0 (0/12), and ypStage 0 . The postoperative course was uneventful, and adjuvant chemotherapy was not administered.

\section{Discussion}

The true clinical benefit of preoperative chemotherapy in the treatment of LACC is yet to be determined [3]. Preoperative chemotherapy has the potential to decrease the rate of multivisceral surgery and improve the rate of R0 resection. In contrast, there is concern regarding the possible tumor growth during preoperative chemotherapy leading to perforation, bleeding, and obstruction [2]. However, preoperative chemotherapy for LACC with more effective 


\begin{tabular}{ll} 
Case Reports & \multicolumn{2}{c}{ Case Rep Oncol 2021;14:1497-1504 } \\
\cline { 2 - 2 } DOI: 10.1159/000519470 & $\begin{array}{l}\text { @ } 2021 \text { The Author(s). Published by S. Karger AG, Basel } \\
\text { whw.karger.com/cro }\end{array}$ \\
\cline { 2 - 2 } in Oncology & Yoshida et al.: pCR Using Pembrolizumab in Advanced Ascending Colon Cancer
\end{tabular}

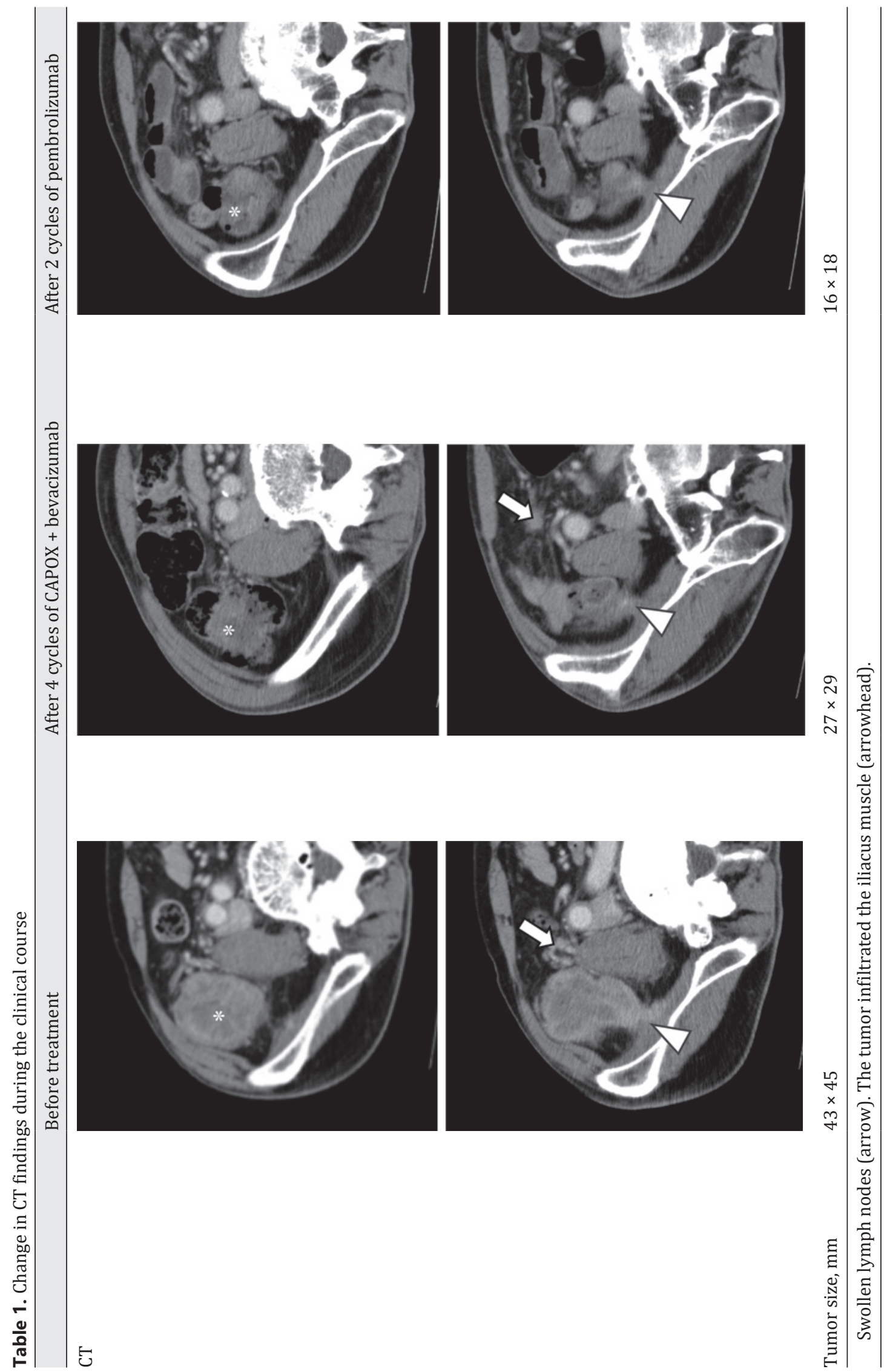

Karger' 


\section{Case Reports in Oncology}

\begin{tabular}{l|l}
\hline Case Rep Oncol 2021;14:1497-1504 \\
\hline DOI: 10.1159/000519470 & $\begin{array}{l}\text { ○ 2021 The Author(s). Published by S. Karger AG, Basel } \\
\text { www.karger.com/cro }\end{array}$ \\
\hline
\end{tabular}

Yoshida et al.: pCR Using Pembrolizumab in Advanced Ascending Colon Cancer

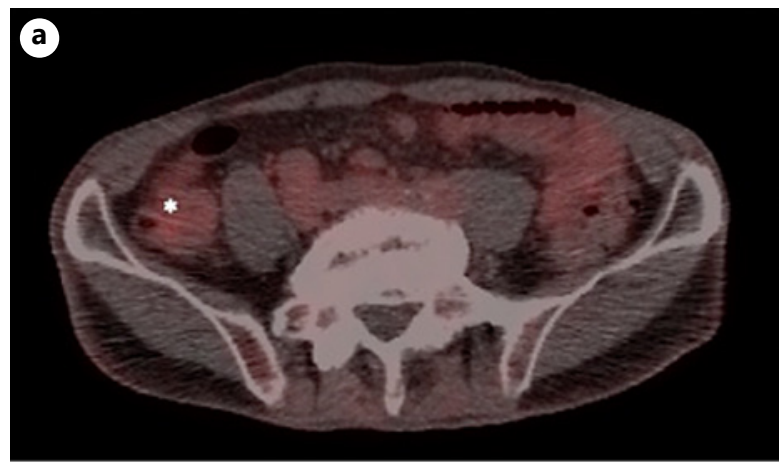

Fig. 2. PET-CT after chemotherapy showed no FDG uptake. FDG, fluorodeoxyglucose; PET-CT, positron emission tomography-computed tomography.
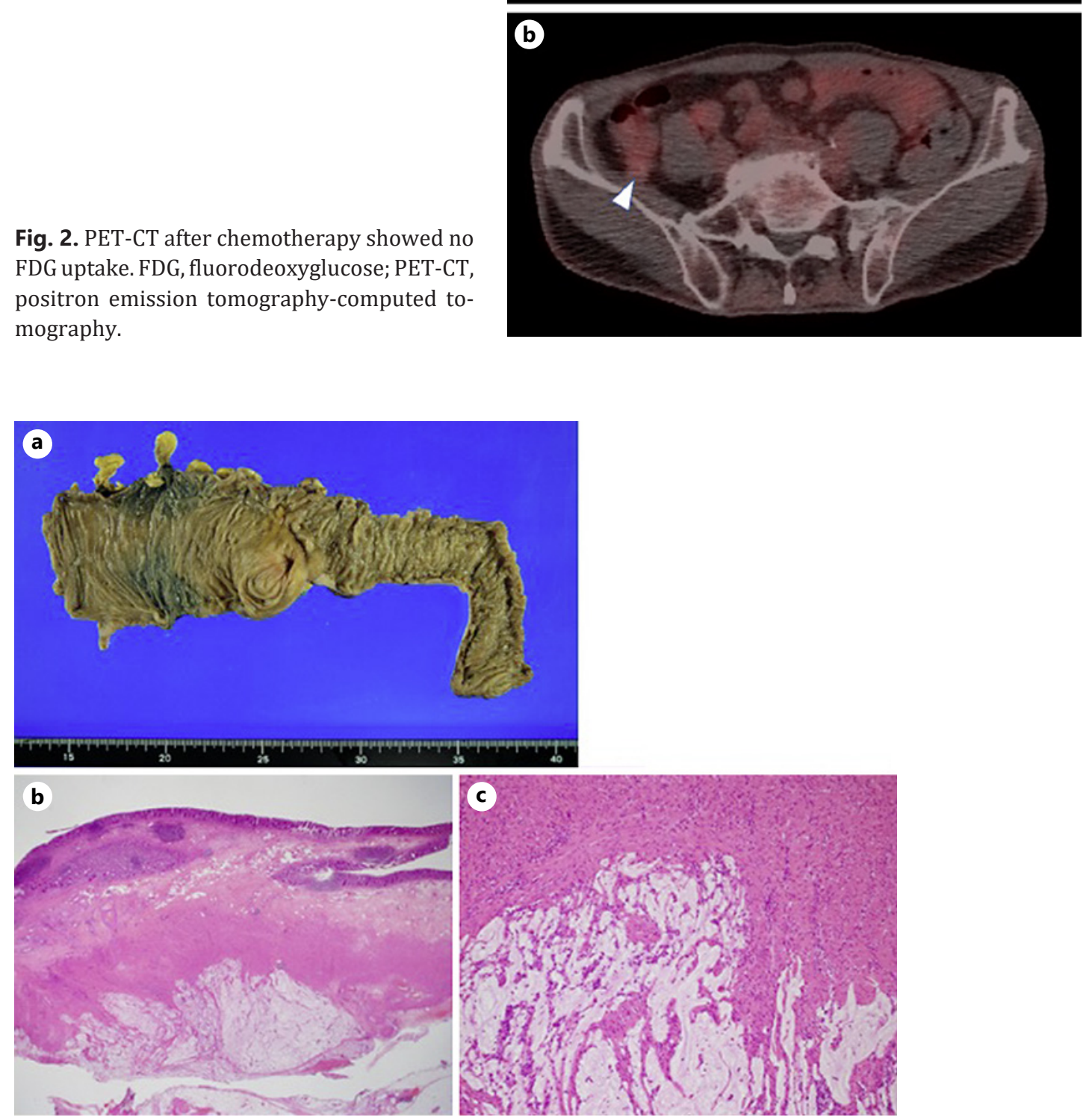

Fig. 3. Gross findings of the resected specimen. No tumor (a). Hematoxylin and eosin (H\&E) stain. No residual cancer cells, and accumulation of mucus at the submucosal to subserosa layer in the resected specimen (b) $\times 40$; (c) $\times 100$. 

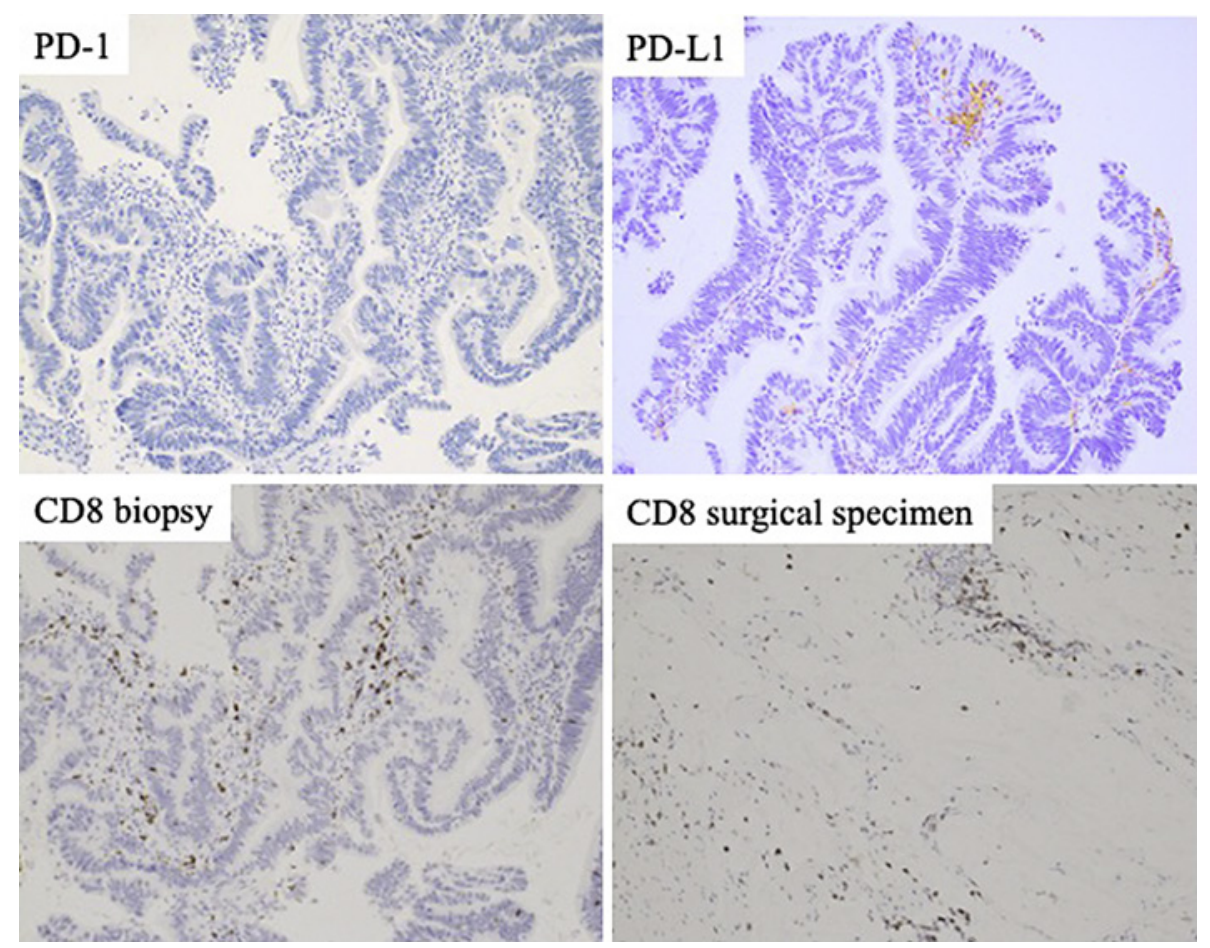

Fig. 4. Immunostaining of the biopsy specimen. Upper left: PD-1, upper right: PD-L1. Immunostaining for CD8. Lower left: biopsy, lower right: surgical specimen. PD-1, programmed cell death 1; PD-L1, programmed cell death-ligand 1.

regimens is currently viewed as a promising option. PD-1/PD-L1 blockade has exhibited substantial antitumor activity in melanoma [4,5], lung cancer [6-8], renal cancer [9], and MSI-H metastatic colon cancer $[10,11]$. Le et al. [12] reported that pembrolizumab led to a $53 \%$ response in 12 different tumor types with MSI-H. Impressive pathological responses to preoperative treatment with immune checkpoint inhibition drugs have recently been demonstrated in early-stage melanoma, lung cancer, and bladder cancer [13-16]. However, few studies have described the use of immune checkpoint inhibition agents, such as pembrolizumab, in preoperative chemotherapy for LACC. The KEYNOTE-177 trial showed that pembrolizumab was superior to standard chemotherapy in MSI-H metastatic colorectal cancer; the risk of disease progression was reduced by $40 \%$, and CR was achieved in $11 \%$ of patients in the pembrolizumab arm. Nevertheless, disease progression occurred in $29.4 \%$ of patients in the pembrolizumab arm; this rate was higher than that noted in the standard chemotherapy arm [17]. Chalabi et al. [18] reported a pCR rate of $60 \%$ in MSI-H nonmetastatic colon cancer patients treated with preoperative ipilimumab/nivolumab with or without celecoxib. Pathological analysis of samples obtained from these patients revealed mixed inflammatory infiltrates, fibrous tissue, and pools of acellular mucin. In posttreatment surgical specimens of deficient mismatch repair tumors, there were significant increases in $\mathrm{CD}^{+}$and $\mathrm{CD}^{+}$cells compared with pretreatment biopsies.

In the present case, CT showed partial response after 4 cycles of CAPOX plus bevacizumab and 2 cycles of pembrolizumab. Pembrolizumab was effective; nevertheless, owing to the residual iliacus muscle invasion detected through $\mathrm{CT}$, we selected operation rather than continued chemotherapy to avoid missing the timing of resection. Consistent with previous findings, pathological examination revealed a CR and mucus accumulation at the submucosal to subserosa layer in the resected specimen (Fig. 3). However, there was no significant difference in the number of $\mathrm{CD}^{+}$cells between the posttreatment surgical specimens and pretreatment 
biopsies. In the present case, the tumor cells disappeared completely and were replaced by mucus and fibrosis. The total lack of tumor cells may explain our inability to accurately assess the abundance of $\mathrm{CD}^{+}$cells. Despite the absence of PD-L1 and PD-1 expression in the pretreatment biopsies, a pCR was achieved. The necessity to evaluate the local environment is unclear because MSI-H colon cancer is expected to benefit from treatment with anti-PD- 1 antibodies; these agents may be effective even in the absence of local expression of PD-1 or PD-L1. Despite the absence of residual cancer cells, it was difficult to confirm a CR using CT because the space previously occupied by the tumor remained filled by accumulated mucus. We suggest that cases judged as "non-CR" in the KEYNOTE-177 study may be similar to the present case, in which the achievement of CR was masked by the accumulation of mucus in the space left by the tumor.

\section{Conclusion}

In this article, we present a rare case of a patient with surgically resected MSI-H advanced ascending colon cancer, in whom an impressive pCR was achieved using preoperative chemotherapy (CAPOX plus bevacizumab, followed by pembrolizumab). The success in the present case may indicate that preoperative chemotherapy using immune checkpoint inhibitors is a useful option for MSI-H LACC.

\section{Statement of Ethics}

The study protocol was approved by the Institutional Review Board for Clinical Research of Kansai Medical University Hospital (2021058). The patient provided informed consent prior to receiving the treatment. The patient also provided written informed consent for the publication of this case report and any accompanying images.

\section{Conflict of Interest Statement}

Hironaga Satake has received research funding from Ono Pharmaceutical Co. Ltd., Daiichi Sankyo, Taiho Pharmaceutical Co., Ltd., Takeda Pharmaceutical Co., Ltd., and Sanofi, honoraria from Bayer Co., Ltd., Bristol-Myers Squibb Co., Ltd., Chugai Pharmaceutical Co., Ltd., Daiichi Sankyo Co., Ltd., Eli Lilly Japan Co., Ltd., Merck Bio Pharma Co., Ltd., MSD Co., Ltd., Ono Pharmaceutical Co., Ltd., Sanofi Co., Ltd., Taiho Pharmaceutical Co., Ltd., Takeda Co., Ltd., and Yakult Honsha Co., Ltd. The other authors have no conflicts of interest to declare.

\section{Funding Sources}

This research did not receive any specific grant or funding from agencies in the public or not-for-profit sectors.

\section{Author Contributions}

Yoshida T., Miki H., and Satake H. contributed to conception and design of the study, Miki H., Kobayashi T., and Matsumi Y. contributed to acquisition of data, Yoshida T., Miki H., Satake H., and Ishida M. contributed to analysis and/or interpretation of data, Yoshida T. and 
Miki H. contributed to drafting of the manuscript, Yoshida T., Satake H., Shibata N., Boku S., Hamada M., and Sekimoto M. contributed to critical review of the manuscript for important intellectual content.

\section{Data Availability Statement}

All data generated or analyzed during this study are included in this article. Further enquiries may be directed to the corresponding author.

\section{References}

1 Lehnert T, Methner M, Pollok A, Schaible A, Hinz U, Herfarth C. Multivisceral resection for locally advanced primary colon and rectal cancer: an analysis of prognostic factors in 201 patients. Ann Surg. 2002 Feb;235(2): 217-25.

2 Croner RS, Merkel S, Papadopoulos T, Schellerer V, Hohenberger W, Goehl J. Multivisceral resection for colon carcinoma. Dis Colon Rectum. 2009 Aug;52(8):1381-6.

3 O'Connor ES, Greenblatt DY, LoConte NK, Gangnon RE, Liou JI, Heise CP, et al. Adjuvant chemotherapy for stage II colon cancer with poor prognostic features. J Clin Oncol. 2011 Sep 1;29(25):3381-8.

4 Robert C, Long GV, Brady B, Dutriaux C, Maio M, Mortier L, et al. Nivolumab in previously untreated melanoma without BRAF mutation. N Engl J Med. 2015 Jan 22;372(4):320-30.

5 Schachter J, Ribas A, Long GV, Arance A, Grob JJ, Mortier L, et al. Pembrolizumab versus ipilimumab for advanced melanoma: final overall survival results of a multicentre, randomised, open-label phase 3 study (KEYNOTE-006). Lancet. 2017 Oct 21;390(10105):1853-62.

6 Borghaei H, Paz-Ares L, Horn L, Spigel DR, Steins M, Ready NE, et al. Nivolumab versus docetaxel in advanced nonsquamous non-small-cell lung cancer. N Engl J Med. 2015 Oct 22;373(17):1627-39.

7 Brahmer J, Reckamp KL, Baas P, Crinò L, Eberhardt WE, Poddubskaya E, et al. Nivolumab versus docetaxel in advanced squamous-cell non-small-cell lung cancer. N Engl J Med. 2015 Jul 9;373(2):123-35.

8 Herbst RS, Baas P, Kim DW, Felip E, Pérez-Gracia JL, Han JY, et al. Pembrolizumab versus docetaxel for previously treated, PD-L1-positive, advanced non-small-cell lung cancer (KEYNOTE-010): a randomised controlled trial. Lancet. 2016 Apr 9;387(10027):1540-50.

9 Motzer RJ, Escudier B, McDermott DF, George S, Hammers HJ, Srinivas S, et al. Nivolumab versus everolimus in advanced renal-cell carcinoma. N Engl J Med. 2015 Nov 5;373(19):1803-13.

10 Le DT, Uram JN, Wang H, Bartlett BR, Kemberling H, Eyring AD, et al. PD-1 Blockade in tumors with mismatchrepair deficiency. N Engl J Med. 2015 Jun 25;372(26):2509-20.

11 Overman MJ, Lonardi S, Wong KYM, Lenz HJ, Gelsomino F, Aglietta M, et al. Durable clinical benefit with nivolumab Plus Ipilimumab in DNA mismatch repair-deficient/microsatellite instability-high metastatic colorectal cancer. J Clin Oncol. 2018 Mar 10;36(8):773-9.

12 Le DT, Durham JN, Smith KN, Wang H, Bartlett BR, Aulakh LK, et al. Mismatch repair deficiency predicts response of solid tumors to PD-1 blockade. Science. 2017 Jul 28;357(6349):409-13.

13 Blank CU, Rozeman EA, Fanchi LF, Sikorska K, van de Wiel B, Kvistborg P, et al. Neoadjuvant versus adjuvant ipilimumab plus nivolumab in macroscopic stage III melanoma. Nat Med. 2018 Nov;24(11):1655-61.

14 Forde PM, Chaft JE, Smith KN, Anagnostou V, Cottrell TR, Hellmann MD, et al. Neoadjuvant PD-1 blockade in resectable Lung Cancer. N Engl J Med. 2018;378:1976-86.

15 Huang AC, Orlowski RJ, Xu X, Mick R, George SM, Yan PK, et al. A single dose of neoadjuvant PD-1 blockade predicts clinical outcomes in resectable melanoma. Nat Med. 2019 Mar;25(3):454-61.

16 Powles T, Kockx M, Rodriguez-Vida A, Duran I, Crabb SJ, Van Der Heijden MS, et al. Clinical efficacy and biomarker analysis of neoadjuvant atezolizumab in operable urothelial carcinoma in the ABACUS trial. Nat Med. 2019 Nov;25(11):1706-14.

17 André T, Shiu KK, Kim TW, Jensen BV, Jensen LH, Punt C, et al. Pembrolizumab in microsatellite-instabilityhigh advanced colorectal cancer. N Engl J Med. 2020 Dec 3;383(23):2207-18.

18 Chalabi M, Fanchi LF, Dijkstra KK, Van den Berg JG, Aalbers AG, Sikorska K, et al. Neoadjuvant immunotherapy leads to pathological responses in MMR-proficient and MMR-deficient early-stage colon cancers. Nat Med. 2020 Apr;26(4):566-76. 